\title{
Huge congenital cystic lung lesion mimicking tension pneumothorax in a child
}

\author{
Ning Wang, M.D. ${ }^{a}$, Xuedong Wu, M.D. ${ }^{a}$, Shanshan Zhang, M.S. ${ }^{a}$ and Na Li, M.S. ${ }^{a}$
}

\begin{abstract}
Treats of symptomatic infants with congenital cysticlung lesions (CCLLs)need surgical resection. Variable location and size of the air cyst of involved lung mean that different surgical strategies must be selected. The presence of huge CCLLs mimicking tension pneumothorax would hamper accurate judgment and timely treatment in dealing with respiratory emergencies. Rare experience reports are coping with childhood tension cystic lung lesion. We present a case of childhood huge CCLLs which was similar to tension pneumothorax based on clinical manifestations and imaging evaluations. Implementation of instant decompression measure against tension pneumothorax failed to alleviate respiratory distress of child. We should exclude the possibility of huge lung cystic lesion in children when clinical evidence inclines to the diagnosis of tension pneumothorax.
\end{abstract}

Key words: lung diseases, congenital abnormalities, cysts, pneumothorax, hospitalized child.

http:/ / dx.doi.org/10.5546/ aap.2019.eng.e416

To cite: Wang N, Wu X, Zhang S, Li N. Huge congenital cystic lung lesion mimicking tension pneumothorax in a child. Arch Argent Pediatr 2019;117(4):e416-e419.

\section{INTRODUCTION}

The treatment strategy of congenital cystic lung lesions (CCLLs) remains controversial. ${ }^{1,2}$ Surgical resection remains a choice for symptomatic infants with CCLLs. ${ }^{3}$ Respiratory symptoms combined with imaging positive findings help pediatricians to identify symptomatic infants with CCLLs. However, vast cystic lung lesion characterized by respiratory distress and special

a Department of Pediatric Surgery, the First Affiliated Hospital, Dali University, Yunnan, China.

E-mail address:

Ning Wang, M.D.: wn20153240@163.com

Funding: None.

Conflict of interest: None.

Received: 9-3-2018

Accepted: 2-11-2019 imaging changes resulting from large space occupying lesion in the thoracic cavity causes difficulties in identifying tension pneumothorax (TP). We present a symptomatic infant with huge CCLLs mimicking TP that perplexed us in coping with respiratory emergency of child.

\section{CASE REPORT}

An 11-month-old boy with respiratory distress and dry cough lasting for three days presented to the pediatric emergency department. The mother had no prenatal control and the child born full term and apparently healthy. His parents denied any episodes of wheeze, history of trauma, congenital diseases, and tuberculosis contact history. He was a thin boy and failed to thrive. His vital signs were the following: respiratory rate, 38 breaths per minute; pulse rate, 130 beats per minute; blood pressure, $82 / 40 \mathrm{mmHg}$; body temperature, $37.2{ }^{\circ} \mathrm{C}$; and finger pulse oxygen saturation, $82 \%$.

The boy had dyspnea and orthopnea. On the physical examination, the thorax on the right side was hyperinflated with the enlargement of the intercostal space and disappearance of breath sound. Rapid radial pulses appeared two-sided. Arterial blood gas analysis showed: $\mathrm{PaO}_{2}, 57 \mathrm{mmHg} ; \mathrm{PaCO}_{2}, 41 \mathrm{~mm} \mathrm{Hg} ; \mathrm{HCO}_{3}^{-}$, $23 \mathrm{mmol} / \mathrm{L}$; BE, $-4 \mathrm{mmol} / \mathrm{L} ; \mathrm{pH}, 7.35$ at room temperature. Chest computed tomography (CT) scan showed a fully compressed right lung and clear viscera (including the trachea, pericardium, and mediastinum) leftward translocation (Figure 1 a1, a2). Radiologist on duty warned the presence of tension pneumothorax. The primary diagnosis was tension pneumothorax.

The boy was immediately taken to the operation room for tube thoracostomy under general anesthesia. A 26Fr chest tube was inserted in the second intercostal space at the midclavicular line. Unfortunately, tachypnea and cyanosis of the boy were still existing after placing a chest tube. Fluctuation of fluid level in the sealed water bottle was weak, and no bubble's overflow arose. Sequential CT scan confirmed the correct position of the chest tube. Meanwhile, a 
suspected giant bulla presented in the right chest, and the right lung condensed (Figure $1 b 1, b 2$ ). The exploratory thoracotomy was performed under general anesthesia. Giant air cyst originated from the superior lobe of right lung nearly occupied the entire cavity. The chest tube is located outside the air cystic lesion. Cyst was excised completely, and the lung recruitment as well. After second surgical intervention, the acute respiratory distress symptoms disappeared and hypoxemia corrected. Pathological reports revealed congenial cystic degeneration of right lung. On day 3 and 7, the following CT checks indicated reducing gas volume and expansion of impaired lung (Figure $1 c, d$ ). In day 11, the boy was discharged without other complications. During a three-year follow-up, the boy had not undergone recurrent pneumonia, cough, dyspnea or other related complications. The physical and nutrition status of the child has improved significantly.

\section{DISCUSSION}

CCLLs are an uncommon developmental malformation of respiratory terminal airway replaced by over-proliferating and dilating multicystic areas. ${ }^{3}$ The incidence rate of CCLLs is between 1 in 10,000 to 1 in 35,000, and most of CCLLs are found on antenatal ultrasound in 20-week gestation. ${ }^{4}$

Treatment strategies for CCLLs remain controversial as poor understanding in etiology and evolution of the disease. Surgical resection once was a mainstream choice for treating CCLLs. Canada pediatric surgeons responded that $80 \%$ neonates with CCLLs were ultimately undergone surgical resection. ${ }^{5}$ In most pediatric surgical centers worldwide, the implementation of the surgical resection did not take into account the

FIGURE 1. Chest computed tomography in preoperative and postoperative stages of the case. a1-a2, images of cupula pleurae and trachea carina layer before chest drainage. b1-b2, images of cupula pleurae and trachea carina layer after chest drainage. $c-d$, on day 3 and 7 , images of chest after thoracotomy.
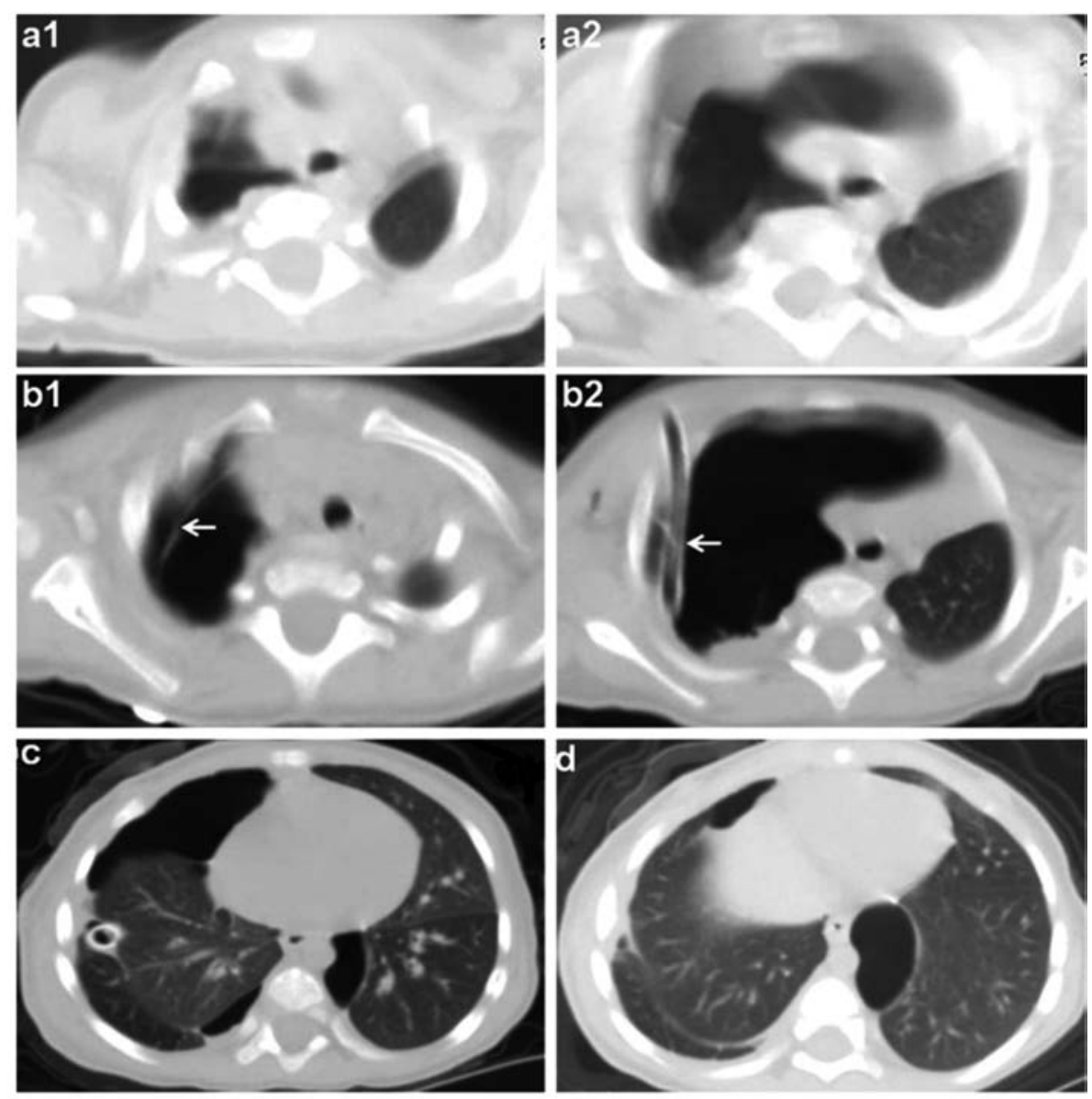
size of the cysts and the symptoms of the patients. ${ }^{6}$ Further studies try to screen out proper patients who need surgical intervention. Symptomatic patients with CCLLs failed to the response to medical treatment were recommended to receive surgical excision. ${ }^{7}$ Asymptomatic patients with CCLLs are advised to take nonoperative treatment. ${ }^{8}$ Although there is no consensus on surgical treatment of CCLLs, symptomatic CCLLs are more likely to need operative resection.

Most of the symptomatic patients with CCLLs need emergency surgery because of worsening of respiratory or hemodynamic condition. ${ }^{6}$ Higher rates of postoperative complications and mortality appear in emergency surgery. ${ }^{6}$ It may relate to instability of respiratory condition of symptomatic patients when emergency surgery performed. Percutaneous transthoracic catheter drainage (PTCD) for large cystic lesion before surgical resection can rescue instability of respiratory condition and reduce the incidence rate of postoperative complications. ${ }^{9}$

CCLLs may accompany with spontaneous pneumothorax (SP). ${ }^{10,11}$ Although SP is uncommon in children, twenty-one percent of pediatric SP are associated with CCLLs. ${ }^{2}$ Both of decompression measure and surgical resection of the cystic lesion are necessary to CCLLs complicated with SP. For CCLLs without pneumothorax, there is a reasonable concern that misdiagnosis of huge CCLLs for a TP would lead to pleural decompression and failure to reduce higher pressure in the large cyst. Decompression measure against TP needs to ensure reducing higher pressure in the pleura cavity. However, PTCD performed under fluoroscopy guidance which tubes or catheters are inserted into the cystic lesion. ${ }^{9}$

Asymptomatic children with undetected CCLLs appear sudden respiratory distress and imaging changes of an enlarge air-containing cyst similarly to clinical features of TP are rare. In the present report, the boy with undetected CCLLs remains symptomless afterbirth until appearance of respiratory distress. A sudden enlargement of an air-filling cystic lesions causes instability of respiratory condition and similar imaging changes in TP. Failure of decompression measure against TP reveals the need for accurate distinction between huge CCLLs and TP.

This case reminds pediatricians to consider the likelihood of happening of huge CCLLs when the pediatric symptoms and imaging findings inclined to the diagnosis of TP. Especially, for asymptomatic children with undetected CCLLs who present clinical features of respiratory emergency similarly to TP, CCLLs should be considered.

\section{REFERENCES}

1. Kapralik J, Wayne C, Chan E, Nasr A. Surgical versus conservative management of congenital pulmonary airway malformation in children: A systematic review and metaanalysis. J Pediatr Surg. 2016; 51(3):508-12.

2. Dotson K, Timm N, Gittelman M. Is spontaneous pneumothorax really a pediatric problem? A national perspective. Pediatr Emerg Care. 2012; 28(4):340-4.

3. Durell J, Lakhoo K. Congenital cystic lesions of the lung. Early Hum Dev. 2014; 90(12):935-9.

4. Lakhoo K. Management of congenital cystic adenomatous malformations of the lung. Arch Dis Child Fetal Neonatal Ed. 2009; 94(1):F73-6.

5. Lo AY, Jones S. Lack of consensus among Canadian pediatric surgeons regarding the management of congenital cystic adenomatoid malformation of the lung. J Pediatr Surg. 2008; 43(5):797-9.

6. Stanton M, Njere I, Ade-Ajayi N, Patel S, Davenport M. Systematic review and meta-analysis of the postnatal management of congenital cystic lung lesions. J Pediatr Surg. 2009; 44(5):1027-33.

7. Parikh D, Samuel M. Congenital Cystic Lung Lesions: Is Surgical Resection Essential? Pediatr Pulmonol. 2005; 40(6):533-7.

8. Ng C, Stanwell J, Burge DM, Stanton MP. Conservative management of antenatally diagnosed cystic lung malformations. Arch Dis Child. 2014; 99(5):432-7.

9. OhSH, Kim CY, Lee BS, Kim DK, et al. Transthoracic catheter drainage for large symptomatic congenital pulmonary airway malformation. Pediatr Pulmonol. 2017;52(12):1572-7.

10. Attou R, Reper P. Complicated pneumothorax and congenital lung cystic malformation. Acta Clin Belg. 2016; 71(5):313-5.

11. Diaz Pumará E, Mortarini MA. Neumotórax hipertensivo como forma de presentación de una malformación adenomatoidea quística pulmonar. Arch Argent Pediatr. 2013; 111(2):e54-7. 